Florida International University

FIU Digital Commons

4-18-1994

\title{
A comparison study of hip range of motion and electromyography during backward walking between children with spastic diplegia and children without known disabilities
}

Martin L. Carrillo

Florida International University

DOI: $10.25148 /$ etd.FI14052587

Follow this and additional works at: https://digitalcommons.fiu.edu/etd

Part of the Physical Therapy Commons

\section{Recommended Citation}

Carrillo, Martin L., "A comparison study of hip range of motion and electromyography during backward walking between children with spastic diplegia and children without known disabilities" (1994). FIU Electronic Theses and Dissertations. 2057.

https://digitalcommons.fiu.edu/etd/2057 


$$
\begin{gathered}
\text { FLORIDA INTERNATIONAL UNIVERSITY } \\
\text { Miami, Florida }
\end{gathered}
$$

\begin{abstract}
A COMPARISON STUDY OF HIP RANGE OF MOTION AND ELECTROMYOGRAPHY DURING BACKWARD WALKING BETWEEN CHILDREN WITH SPASTIC DIPLEGIA AND CHILDREN WITHOUT KNOWN DISABILITIES
\end{abstract}

A thesis submitted in partial satisfaction of the requirements for the degree of

$$
\begin{gathered}
\text { MASTER OF SCIENCE } \\
\text { IN } \\
\text { PHYSICAL THERAPY }
\end{gathered}
$$

by

Martin L. Carrillo 
To: Dean William J. Keppler College of Health

This thesis, written by Martin L. Carrillo, and entitled A COMPARISON STUDY OF HIP RANGE OF MOTION AND ELECTROMYOGRAPHY DURING BACKWARD WALKING BETWEEN CHILDREN WITH SPASTIC DIPLEGIA AND CHILDREN WITHOUT KNOWN DISABILITIES, having been appoved in respect to style and intellectual content, is referred to you for judgement.

We have read this thesis and recommend that it be approved.

Jennifer Lander

Carlos Alvarez

Leonard Elbaum, Major Professor

Date of Defense: April 18, 1994

The thesis of Martin L. Carrillo is approved.

Dean William J. Keppler College of Health

Dr. Richard I. Campbell

Dean of Graduate studies

Florida International University, 1994 
To Melissa, Nicole, Alain, Jonathan, Idelfonso and Adrian, the six children who participated in the study, for allowing us to learn about gait, but most importantly, about life. 


\section{ACKNOWLEDGMENTS}

My deepest appreciation to Professor Leonard Elbaum for the generosity with his knowledge, time, humor and experience.

Thanks to Dr. Jennifer Lander for the invaluable help with the content and style for this project.

My appreciation to Dr. Carlos Alvarez for his participation in the Thesis Committee.

It was a privilege to be involved with such oustanding professionals and human beings. 
A COMPARISON STUDY OF HIP RANGE OF MOTION AND ELECTROMYOGRAPHY DURING BACKWARD WALKING BETWEEN CHILDREN WITH SPASTIC DIPLEGIA AND CHILDREN WITHOUT KNOWN DISABILITIES

by

Martin L. Carrillo

Florida International University, 1994

Miami, Florida

Professor Leonard Elbaum, Major Professor

The purpose of this study was to examine the kinematics of the pelvis and the right hip and the electromyographic activity of the gluteus maximus and biceps femoris during backward walking in children with spastic diplegic cerebral palsy and children without known disabilities. Subjects included 6 children, three with a diagnosis of spastic diplegia and three without known disabilities.

The results of the study showed important differences between the experimental group and the control group. Subjects with spastic diplegic cerebral palsy (SDCP) walked backwards with less hip extension, more body rotation, shorter and slower steps, and used a higher percentage of their maximum voluntary contraction than the control group. No significant 
differences were found between both groups in the total amount of hip sagittal range of motion; however, these motions occurred towards flexion in the group of children with spastic diplegic cerebral palsy and towards neutral and extension in the control group. Independent backward walking seems not to be an effective way to promote active hip extension in children with spastic diplegic cerebral palsy.

Clinicians using backward walking in therapeutic programs may benefit from this data. 


\section{Table of Contents}

\section{Page}

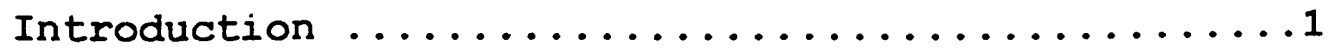
Purpose of the study $\ldots \ldots \ldots \ldots \ldots \ldots \ldots \ldots \ldots$

Review of Literature.................

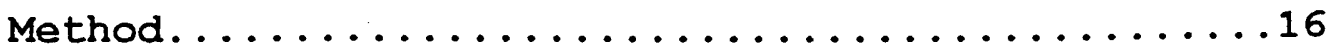

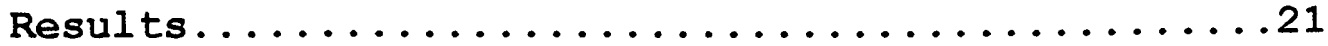

Discussion...........................

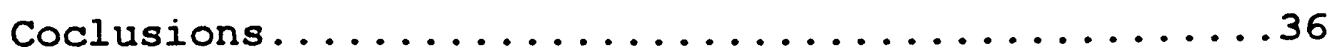

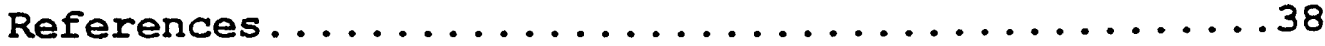




\section{Introduction}

The need for improved research in the field of physical therapy to justify the services provided appears evident. The changes in the American health system, the pressures from the insurance companies, and from Medicaid and Medicare programs are some of the elements that are acting as a catalyst for our profession to review approaches and techniques previuosly considered widely accepted. In the last decade, an increasing number of physical therapists are becoming researchers and scholars and their studies are questioning the bases and rationale of various therapeutic approaches.

Human gait is one of the most popular areas of research in physical therapy and rehabilitation, especially since the development of computerized gait analysis. Although there is abundant literature regarding forward gait, there is scarce information about backward gait. Only one scientific study regarding normal or abnormal backward walking was found (Cipriani, Armstrong, \& Gaul, 1993). A brief comment was written by Bobath (1975 a, pp. 110-111) about the importance of teaching the patient with adult hemiplegia to flex the knee while stepping backwards, correcting the tendency of the patient to lift the pelvis in compensation for the 
difficulty of clearance of the foot. Bobath also recommended the use of backward walking alternately with forward walking in adult patients with post-stroke hemiplegia. Conversations with faculty members of several physical therapy programs in Florida (personal Communications, May, June, August, 1993), a staff of the Motion Analysis Laboratory at Miami Children's Hospital (personal communication, September, 1993) and many professionals in Miami hospitals (personal communications, 1993) did not produce any further information regarding research in backward gait.

Although many therapists teach backward gait to their patients, most are not aware of any research validating the use of this activity. Backward walking, active and/or assisted by the therapist, is a common activity used by therapists with their neurologically injured patients. During informal conversations with many physical therapists, several reasons were obtained to explain the clinical use of backward walking:(a) the need to teach it as a milestone in the child's normal development (Caplan \& Caplan, 1977); (b) the need to teach backward stepping to develop balance and avoid falling; (c) to promote a variety of movement patterns; (d) to promote knee flexion in stiff-legged gait; (e) to 
promote hip extensor activity and hip extensor control in patients with excessive hip flexor posturing or spasticity, and (f) for spatial perceptual training. This author has used backward walking in children with cerebral palsy to facilitate flexor and extensor control of the lower extremities. During backward gait, these patients have difficulties which requires the use of various techniques of manual assistance from the therapist, especially with pelvic and hip alignment, hip extensor and abductor control, knee control and heel contact and control. This assistance, provided externally, should be gradually decreased in order for the patient to develop his/her own motor control. This author has seen many children with spastic diplegia learn to walk backwards independently, as well as with assistance, and one of the motivations of this study was to understand the mechanisms underlying the adquisition of this skill.

Spastic Diplegia is the most common type of cerebral palsy, representing between 328 ( Nelson \& Ellenberg, 1979) and 418 (Hagberg, Hagberg \& VonWendt, 1989) of the total group. It is characterized by motor involvement of the whole body, especially the lower trunk, pelvis and lower extremities (Bobath, 1975 b). Asymmetrical involvement of the body is not uncommon. Strabismus and 
problems with visual contact and tracking are also common in individuals with this condition. The aetiology of spastic diplegia seems to be related to preterm birth and prenatal factors, being periventricular lesions due to intraventricular haemorrhage; one of the most common causes (Aicardi, 1992). 


\section{Purpose of the Study}

The purpose of the study was to examine and compare the kinematics of the pelvis and the hip joint and the electromyographic activity of the gluteus maximus and biceps femoris during backward stepping between children with spastic diplegic cerebral palsy and children without known disabilities. 
Review of Literature

Gait Analysis Studies in Children with Cerebral Palsy

Sutherland (1981) presented one of the first indepth gait analysis studies of children with cerebral palsy. In one boy with spastic diplegia, the electromyographic study was done with motion control MC-1 surface electrodes and revealed premature onset of phasic activity in the gastrocnemius-soleus and in the medial hamstrings and prolongation of activity in the stance phase of the vastus medialis and vastus lateralis. He also found excessive knee flexion and ankle dorsiflexion with strong quadriceps action to maintain stability and deficient push-off force. Being a surgeon, he was looking for surgical solutions, so he summarized the preoperative problem as muscle imbalance of the knee with chronic over-stretching of the quadriceps muscle and patellar tendon and contracture of the hamstrings muscles.

In a case study of a 9 year-old girl with spastic quadriplegia, Sutherland (1982) found a walking velocity of $14 \mathrm{~cm}$. per second (less than $20 \%$ of the normal velocity) and a cadence of 73 steps per minute (about 50\% of the normal cadence). This patient showed a particularly abnormal anterior pelvic tilt, limited knee 
flexion in swing phase, marked equinus during the entire cycle, and severe restriction of the single limb stance time, spending most of the time in double stance. The EMG study showed a rectus femoris active through all the swing phase, interfering with the normal knee flexion. Medial hamstrings, rectus femoris and vastus lateralis showed phasic prolongation in stance phase. Phasic prolongation was also recorded in the gastrocnemiussoleus and tibialis posterior, as well as premature onset of gastrocnemius-soleus.

In an attempt to assess the outcome of surgical procedures, Gage, Fabian, Hicks, and Tashman (1984) presented a study of pre and post-operative computerized gait analysis in 20 children with spastic diplegia. Their most important finding was that out of 272 muscles investigated pre and post-operatively, 249 showed unchanged phasic activity. In the 23 that did change, no trends were seen. A comnon EMG finding was the simultaneous firing of quadriceps and hamstrings at midswing phase, a period when they would normally be inactive. This high percentage of unchanged phasic activity after surgery has generated some controversy about the effectiveness of surgical, therapeutic and orthotic procedures in individuals with central nervous system disorders. 
Tylkowski, Howell-Garvey, and Miller (1988) presented preliminary information regarding the influence of the abnormal motor activity of the hamstrings and hip flexors in the gait of children with cerebral palsy. These patients were clustered into three primary groups based on pelvic motion during the gait cycle, showing decreased velocity with increased abnormality of pelvic motion from group I to group III. Group I showed a crouch gait caused by hip flexor contracture with anterior pelvic tilt, out of phase gluteus maximus muscle activity, and occasional prolonged hamstring activity. Group II was a crouch gait caused by hamstring contracture showing posterior pelvic tilt with prolonged hamstring activity. Group III was a group of patients with a crouch gait caused by a combination of hip flexor and hamstring contracture resulting in a more normally tilted pelvis, with abnormal hamstrings activity and occasional out of phase gluteus maximus muscle activity. These authors emphasized the importance of the study of pelvic motion and dynamic EMG to delineate more accurately the specific contributions of hamstring and hip flexor musculature to spastic crouch gait.

Tylkowski and Howell (1990) presented a gait analysis study of an 11-year-old with spastic diparesis and right hemiparesis. They found that the patient 
had decreased velocity, stride, and step lengths. There was asymuetry of the step length with the left step significantly longer that the right; slightly decreased cadence secondary to shortened stride length; and increased time spent on double support and on his less affected lower extremity. The left hip flexion was diminished in motion, achieving only 30 degrees of flexion and remaining in 10 degrees of flexion during stance. The pelvic rotation was asymetrical with the right side remaining further forward. The EMG analysis showed abnormal activity of the left hamstrings during single limb stance and early weight release. Abnormal activity was also noted in the rectus femoris during the swing phase. The left hip adductor musculature was active through almost the entire cycle while gluteus medius was active through all of stance and the beginning of the swing phase.

In a more recent study performed by Tylkowski and Howell(1993) with a 5-year-old girl with spastic diplegia, the findings were: the subjects's hips were unable to reach full extension in stance, gluteus maximus over-active during stance phase, and a pelvis anteriorly tilted. The study also suggested that the anterior pelvic tilt, in spite of hamstring interference, may be caused by shortening of hip flexor musculature. 
Another interesting finding was that which

related to the asymmetries of pelvis and trunk and their impact on the kinematics of the hips. The right side of the trunk and pelvis remained forward and the right side of the pelvis was elevated. Her trunk was leaning to the left side. She also had a very diminished rotation of the trunk and pelvis, moving these two segments as a unit. The study suggested that the pelvic asymetry produced a right hip adduction and left hip abduction during stance, while in swing both tended to increase in abduction. The rotational asymetry of the pelvis did not match the hip position, with both hips externally rotated, more the left than the right. This patient's gait was very slow, with a speed of about $13.9 \mathrm{~cm} / \mathrm{sec}, 12.88$ of the normal and a cadence of 58.1 steps $/ \mathrm{min}$, about $37.7 \%$ of the normal.

The contrasting effects of the use of posterior and anterior walkers in the gait parameters of children with cerebral palsy has been studied by several researchers including Greiner (1993), Logan, Byers-Hinkley, and Ciccone (1987). Their data suggested that the use of posterior walkers facilitated a more upright posture, decreasing the hip and trunk flexion during stance phase, decreasing the double stance time and increasing the walking velocity. Howell-Garvey, Tylkowski, Kates 
and Miller (1987) presented a study suggesting that the use of a posterior walker produces changes in shoulder, trunk, pelvis positions and hip extension, but they did not find a translation of these changes in objective functional improvements.

Many surgical procedures have been designed and implemented in children with cerebral palsy, including the hip and knee musculature. Nene, Evans, and Patrick (1993) presented data from a study of 19 children with simultaneous multiple operations. In 15 of 17 patients, an intrapelvic intramuscular psoas tenotomy produced improvement of the hip flexion deformity without the loss of muscle power to initiate the swing phase. Also, the distal transfer of the rectus femoris when it was found to be contracting inappropriately, a surgical procedure originally proposed by Jacquelin Perry, improved the knee flexion during walking from a mean of 28.3 degrees to 45.2 degrees.

\section{Electromyographic Studies}

The electromyogram (EMG) has been considered the single best representation of the neurological activation of the skeletal muscle (Winter, 1987). The use of surface electromyography in gait studies is very convenient since the application is simple and is relatively painless 
(Perry \& Antonelli, 1993). The use of surface electrodes is appropriate if the objective is to obtain information about gross phasing activity or interval or magnitude of peak muscle activity. When the objective is to investigate accurate timing of muscle action, Perry and Antonelli prefer the use of fine wire electrodes. The area of "cross-talk," which may contain low amplitude signals from other muscles in the vicinity of the one being investigated, should not be given functional significance, therefore Perry and Antonelli proposed to delete the lowest $15 \%$ of the EMG signal to avoid it. The performance of surface and intramuscular wire electrodes in gait analysis was studied by Kadaba, Wooten, Gainey, and Cochran (1985). They found that data recorded from surface electrodes represents a more constant and reliable measure of muscle activity, tested on different test days, than data recorded from the commonly used bipolar intramuscular wire electrodes.

Some of the parameters that may affect the magnitude and quality of the EMG signal are: electrode location, electrode size and shape, spacing between electrodes, fat tissue overlying the muscle, muscle temperature, and cross sectional area and length (Winter, 1987).

In order to record electromyographic activity the surface electrodes are placed over the motor points. 
Muscle motor points are areas of low threshold of excitability, approximately perpendicular to the main direction of the muscle fibers. They do not necessarily correspond to the site of nerve entrances into the muscles, but rather to the region where there is a great density of terminal elements near the surface (Walthard K.M. and Tchicaloff, 1971). These authors consider the motor points as an anatomical entity and not a physiological property of muscle fiber.

Besides the motor points, the skin has other highly sensitive areas to electrical stimulation. These are areas where the motor nerves are located so superficially that the application of relatively low currents will be transmitted to all muscles supplied distally to the points of stimulation (Walthard K.M. and Tchicaloff, 1971).

The gluteus maximus and the biceps femoris are the two most important hip extensor muscles. The gluteus maximus shows two peaks of activity during normal forward gait. The first at the beginning of the of the loading phase, right after heel contact, and the second peak at the end of the swing phase. According to Basmajian and De Luca (1985) the gluteus maximus activity is to prevent or to control flexion at the hip joint.

Biceps femoris activity is similar to the gluteus 
maximus musculature with two periods of activity: one immediately after heel contact and one in the middle of the swing phase (Sutherland, 1982).

Backward Walking

Backward walking at three levels of treadmill inclination was studied by Cipriani, Armstrong, and Gaul (1993), on sixteen randomly selected healthy adult subjects. This study evaluated the changes in hip, knee, and ankle kinematics, as well as in the EMG activity of hamstrings, rectus femoris, gastrocnemius, and tibialis anterior, by walking backwards at 0 degrees, 5 degrees and 10 degrees of platform inclination. These authors found significant increase in EMG activity of hamstrings, rectus femoris, anterior tibialis and gastrocnemius at 10 degrees of platform inclination, and they suggest inclusion of backward walking on a treadmill as an adjunct to lower extremity rehabilitation.

In summary, the review of literature regarding gait EMG studies in children with spastic diplegic cerebral palsy showed the following: premature onset or prolongation of hamstring activity, simultaneous firing of hamstrings and quadriceps at midswing phase and asymmetrical activity of several muscles of the lower 
extremity due to uneven involvement in both sides of the body. The gait kinematics studies of children with spastic diplegic cerebral palsy showed decreased and asymmetrical step length, decreased walking velocity, decreased cadence, and decreased single limb stance. The gait kinematics studies showed that the hip had decreased extension in stance, increased flexion in swing, increased adduction, increased internal rotation, and asymmetries between the right and the left hips. The pelvis showed increased anterior tilt, increased posterior tilt and asymetrical frontal and horizontal rotations. No studies or reports showing benefits of practicing backward walking in children with spastic diplegic cerebral palsy or in children without known disabilities were found.

Backward walking is an activity commonly included in therapeutic programs with children with spastic diplegic cerebral palsy. The purpose of this study is to examine and compare the EMG activity of gluteus maximus and biceps femoris and the kinematics of the pelvis and right hip between a group of children with spastic diplegic cerebral palsy and a group of children without known disabilities. 
Method

This is a descriptive pilot study designed to examine and compare kinematics and EMG data between and experimental and a control group.

Subjects

The subject group of the study consisted of six children, three with a diagnosis of spastic diplegic cerebral palsy (experimental group) and three without known disabilities (control group), chosen from the author's private patients and his acquaintances. In the experimental group, one of the subjects was an independent ambulator and the other two required the use of posterior walkers. They were sex matched and approximately age matched. They were examined via surface EMG and computer assisted videography while backward walking. The examination was limited to one hip in each subject. The age range of both groups was from 3 to 12 years. All participants were tested in the same room and on the same walking platform at the motion analysis laboratory of the physical therapy program, at Florida International University, Miami, Florida. Informed consent was obtained from the parents or guardians of every child tested. Children were evaluated in the presence of their parents, guardians or relatives. 
Equipment and EMG Placement

Subjects were tested using the Ariel Performance Analysis System which is a computerized program that processes information from force plate, EMG and videography. The EMG activity was assessed with bipolar silver-silver chloride electrodes, and two Sony industrial quality video cameras recorded bidimensional images of the subjects' hip kinematics. Subjects were marked with reflective markers for the kinematics studies, which were placed at the right shoulder, right anterior superior iliac spine (ASIS), right posterior iliac spine (PSIS), and at the right femoral condyle. Electrodes for the electromyographic study were placed at the motor points for the right gluteus maximus and biceps femoris. The gluteus maximus muscle is supplied by the Inferior Gluteal nerve (L5, S1, S2) (Lockhart, Hamilton, \& Fyfe, 1965) and has 3 motor points, one located about the center of the lower inner quadrant of the buttock and the other two motor points near the center of the buttocks. For this study, the middle motor point was used. The biceps femoris is supplied by the Sciatic nerve (L4, L5, S1, S2, S3) and has two motor points. One is located in the center of the thigh, about three fingers breadth below the gluteal fold, which was used for 
the examination. The other motor point is located immediately above the knee joint (Walthard and Tchicaloff, 1971).

\section{Procedure}

The following procedure was applied with each subject:

1. The subject and his/her parents/guardians received a clear explanation of the experiment.

2. The subject was asked or assisted to remove shoes, socks and orthoses. He/she was wearing a bathing suit.

3. With the parents or guardians present, the subject assumed the prone position on a treatment table.

4. The area of the skin over the gluteus maximus and biceps femoris musculature was cleaned with alcohol.

5. The electrodes were placed over the motor points as described by Walthard and Tchicaloff (1971). The electrodes were held down with skin tape.

6. The reflective markers were placed at the right shoulder, right ASIS, right PSIS and right femoral condyle.

7. The subject was asked to perform a maximum voluntary contraction (MVC) in three different ways : (a) in prone position he/she was asked to raise the 
thigh; (b) in prone position he/she was asked to avoid the fall of the lower extremity suspended at the edge of the treatment table; (c) in supine position with the lower extremity in flexion, he/she was asked to push against the resistance provided by the examiner. The best response was considered the maximal voluntary contraction (MVC) .

8. The subject was asked or assisted to a standing position and directed towards the walking platform.

9. A demonstration of a person walking backwards was performed for the subject to observe.

10. The subject was asked to walk backward. Verbal encouragement was offered, specially with the group of children with spastic diplegia.

11. The subject walked backwards (approximately 10 feet) a minimum of three times, while EMG and videography was being recorded.

12. The electrodes were removed and the skin was cleaned.

13. The subject and parents/guardians were thanked for their cooperation.

Data Analysis

For the kinematic study, the video recordings of 
each subject were visually examined. The cycle to be analyzed was defined as the period beginning at the moment of the contralateral foot contact (left foot), through backward swing of the right lower extremity, and up to the full contact of the right foot. A typical cycle was chosen. A digital model was created via Ariel Performance Analysis System. Data was collected for the six subjects in pelvic sagittal range of motion (Table 2), maximum posterior tilt and maximum anterior tilt (Table 2), hip saggital range of motion and maximum hip extension (Table 3).

For the electromyographic study, the data from each subject was reviewed and the highest peak was considered the maximum voluntary contraction (Table 1). Highest EMG activity of the gluteus maximus and biceps femoris during backward walking was measured (Table 1). The peak EMG activity during backward walking, divided by the MVC (percentage of MVC), was compared between the subjects of both groups. 
Data including pelvic and hip sagittal

kinematics and surface electromyography of gluteus maximus and biceps femoris was compared between a a group of three children with spastic diplegia (experimental group) and a group of three children without known disabilities (control group). Electromyographic data from one subject of the experimental group was considered "noise or artifact" and was excluded from the study. Maximum Voluntary Contraction (MVC) was not obtained in another subject of the experimental group due to lack of cooperation.

Data obtained and derived from each subject included:

1.EMG during Maximum Voluntary Contraction (MVC) (Table 1)

2. Peak EMG activity during backward walking (Table 1)

3.Peak EMG activity/Percentage of MVC (Table 1)

4. Pelvic sagittal range of motion (Table 2)

5.Maximum Posterior Tilt (Table 2)

6. Maximun Anterior Tilt (Table 2)

7. Hip Sagittal range of motion (Table 3)

8. Maximun Hip Extension (Table 3) 
Table 1

EIECTROMYOGRAPHIC ACTIVITY

MAXIMUM VOLONTARY

CONIRACTION

(MICROVOLTS)
PEAR EMG ACTIVITY DURING BACKWARD

WAIRING (MICROVOLTS)
PEAR EMG ACTIVITY/ MVC (PERCENTAGE)

(z)

$\begin{array}{llllll}\text { GLUTEOS } & \text { BICEPS } & \text { GLUTEUS } & \text { BICEPS } & \text { GLUTEOS } & \text { BICEPS } \\ \text { MAXIMUS } & \text { FEMORIS } & \text { MAXIMOS } & \text { FHMORIS } & \text { MAXIMOS } & \text { FEMORIS }\end{array}$

EXPERTMENTAL

A

6.10

9.20

5.60

4.60

B *

$C \star \star$

CONTROI

$\mathbf{x}$

31.80

40.50

11.90

23.80

7.60

3.10

10.70

3.00

54.90

63.50

928

968

* Subject B did not cooperate with MVC testing.

* * Subject C's EMG was considered noise. 
Table 2

PELVIC MOTION

$\begin{array}{ccc}\text { PELVIC RANGE } & \text { MAXIMOM POSTERIOR } & \text { MAXIMOM ANTERIOR } \\ \text { OF MOTION } & \text { TIIT } & \text { TILT } \\ \text { (DEGREES) } & \text { (DEGREES) } & \text { (DEGREES) }\end{array}$

EXPERIMENTAL
A
4.80
2.80
1.90
B
2.90
$-14.60$
17.50
C
10.20
$-10.30$
20.50

CONTROL

$\begin{array}{rrrr}x & 2.90 & -12.20 & 9.30 \\ y & 2.90 & -23.10 & 20.20 \\ z & 17.00 & 0.00 & 17.00\end{array}$


Table 3

HIP MOTION

$\begin{array}{cc}\text { HIP SAGITTAL } & \text { MAXIMUM } \\ \text { RANGE OF MOTION } & \text { HIP EXTENSION } \\ \text { (degrees) } & \text { (degrees) }\end{array}$

EXPERTMENTAL

$\begin{array}{lll}\text { A } & 30.00 & -2 \\ \text { B } & 27.70 & -22 \\ \text { C } & 10.10 & -52.70\end{array}$

CONTROL

$\mathrm{x}$

$\mathbf{Y}$

z
29.50

25.80

15.30
$-3.20$

$-3.50$

9.20 
Experimental Group

Subject A (a 6-year-old female) showed a maximum voluntary contraction (MVC) of the gluteus maximus of 6.07 microvolts and a value of 5.6 microvolts during backward walking (92 of the MVC). Biceps femoris had a MVC of 9.23 microvolts and 8.83 microvolts during backward walking (96 \& of the MVC). Pelvic range of motion was 4.73 degrees (from 2.81 degrees of posterior tilt to 1.92 of anterior tilt) (Figure 2). The hip range of motion was 30 degrees (from 2 degrees of flexion to 32 degrees of flexion) (Figure 1). Her maximal hip extension was 2 degrees of flexion at the beginning of the cycle (total right foot contact).

Subject B (3-year-old male) had 4.61 microvolts of gluteus maximus activity and 14.13 of biceps femoris activity during backward walking. Due to lack of cooperation no MVC was obtained with this subject. Sagittal pelvic range of motion was 2.89 degrees (from 14.60 degrees of anterior tilt to 17.50 degrees of anterior tilt). Hip range of motion was 27.70 degrees (from 22 degrees of hip flexion to 50.30 degrees of hip flexion). His maximal hip extension was 22 degrees of hip flexion at the beginning of the cycle 


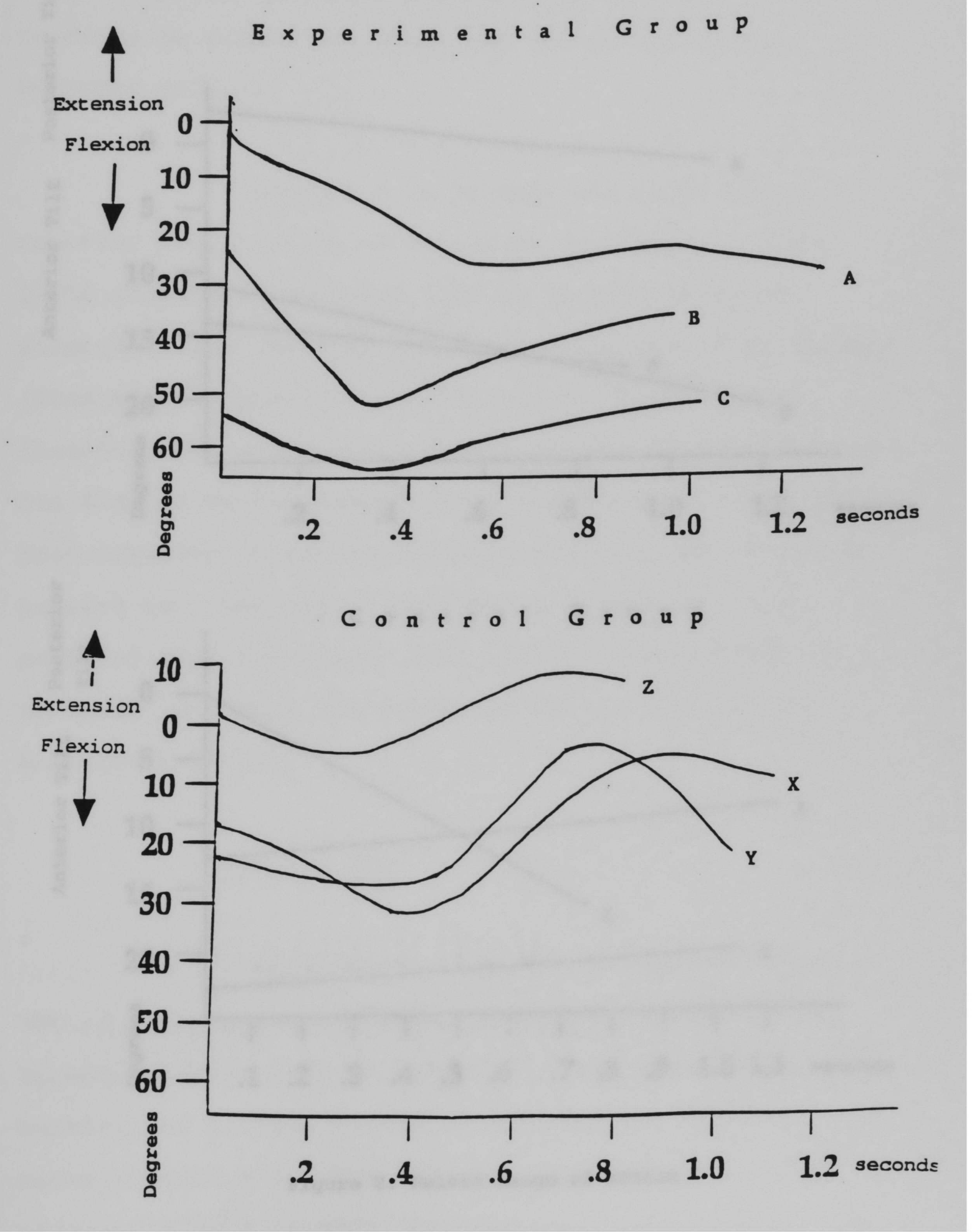

Figure 1. Hip Range of Motion 
+4

E $x p$ e $r i m$ e $n t a l$ G $r$ o $u$

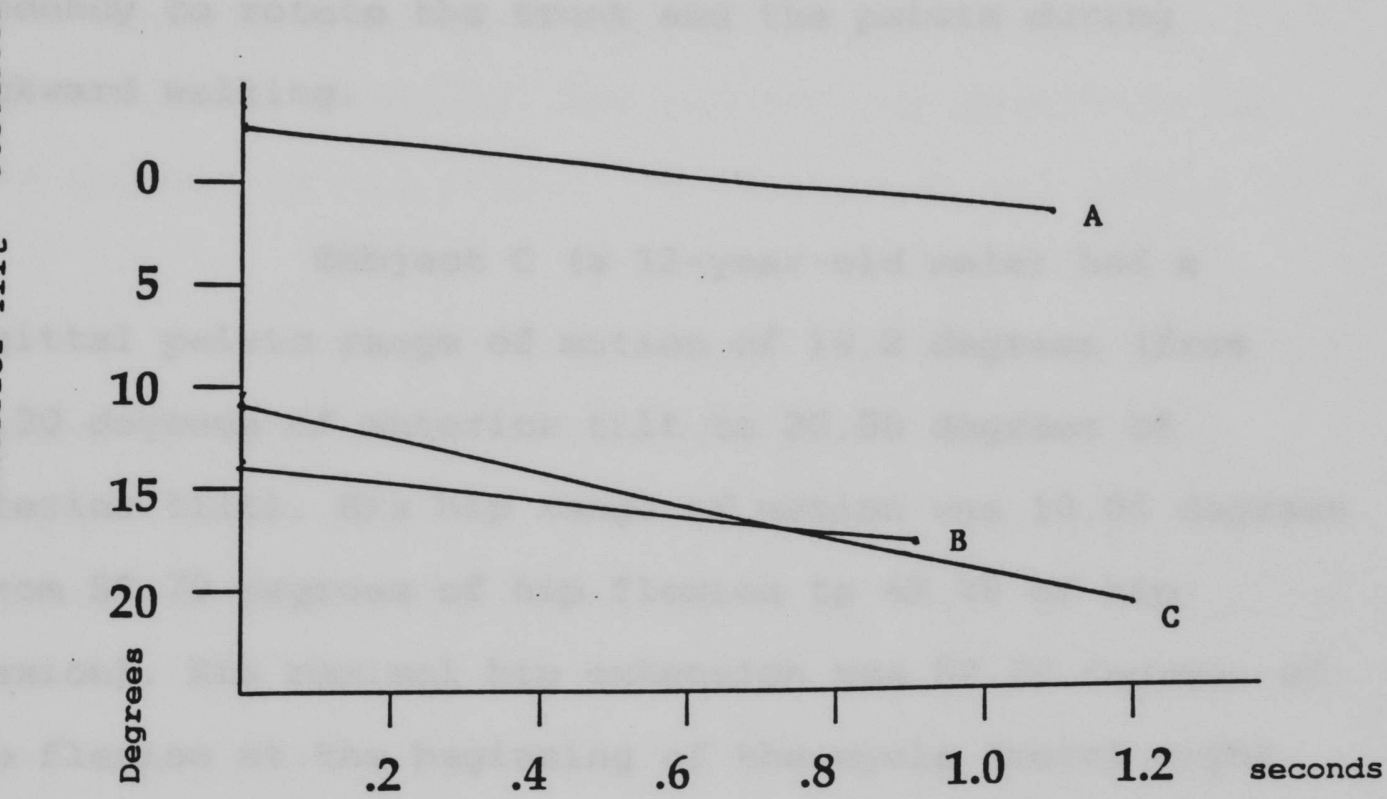

至

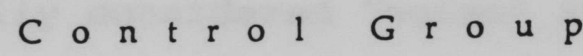

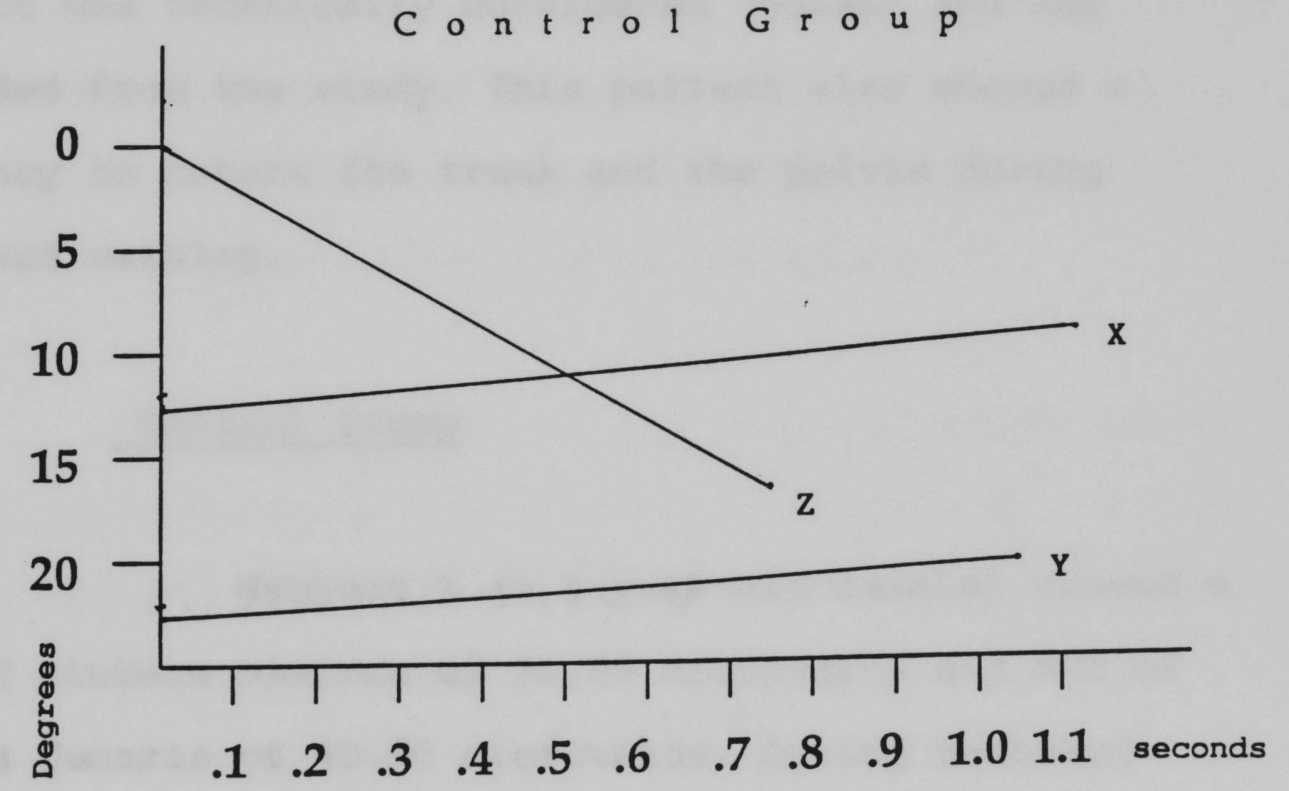

Figure 2. Pelvic Range of Motion 
(total right foot contact). This patient showed a clear tendency to rotate the trunk and the pelvis during backward walking.

Subject C (a 12-year-old male) had a sagittal pelvic range of motion of 10.2 degrees (from 10.30 degrees of anterior tilt to 20.50 degrees of anterior tilt). His hip range of motion was 10.05 degrees (from 52.70 degrees of hip flexion to 62.75 of hip flexion). His maximal hip extension was 52.70 degrees of hip flexion at the beginning of the cycle (total right foot contact). Electromyographic activity data from this patient was technically considered "noise" and was excluded from the study. This patient also showed a tendency to rotate the trunk and the pelvis during backward walking.

Control Group

Subject X (a 6-year-old female) showed a MVC of gluteus maximus of 31.80 microvolts and MVC of biceps femoris of 40.50 microvolts. During backward walking the gluteus showed an activity of 11.90 microvolts ( 37 of $\mathrm{MVC}$ ) and the biceps femoris 16.34 microvolts ( $40 \%$ of $\mathrm{MVC}$ ). Her pelvic range of motion was of 2.93 degrees (from 12.20 degrees of anterior tilt to 
9.27 degrees of anterior tilt). Hip range of motion was 29.48 degrees (from 32.63 degrees of hip flexion to 3.15 degrees of hip flexion). Her maximal hip extension was 3.15 degrees of hip flexion at the end of the cycle.

Subject $Y$ (a 3 year old male) had a MVC of gluteus maximus of 12.62 microvolts and a MVC of the biceps femoris of 23.82. During backward walking the gluteus maximus showed an activity of 7.56 microvolts 160 8 of MVC) and the biceps femoris 3.11 microvolts (13 of of MVC). His pelvis range of motion was 2.94 degrees (from 22.11 degrees of anterior tilt at the beginning of the cycle to 19.17 degrees of anterior tilt at the end of the cycle). His hip range of motion was 25.81 degrees (from 29.28 degrees of flexion during backward swing to 3.47 degrees of flexion at the end of the cycle).

Subject Z (a 9-1/2-year old male) showed a MVC of gluteus maximus of 63.45 microvolts and a MVC of biceps femoris of 54.90 microvolts. During backward walking the gluteus maximus had an activity of 2.99 microvolts ( $5 \%$ of $\mathrm{MVC}$ ) and the biceps femoris 10.68 microvolts (19 8 of MVC). This subject showed a sagittal pelvic range of motion of 17 degrees (from 0 degrees of anterior pelvic tilt to 17 degrees of anterior pelvic tilt). His hip range of motion was 
15.34 degrees (from 6.29 degrees of flexion to 9.15

degrees of hip extension). His maximum hip extension was 9.15 of hip extension at the end of the cycle. 


\section{Discussion}

This study was performed to gather information regarding pelvic and hip kinematics and gluteus maximus and biceps femoris activity during backward walking. The data collected shows important differences between the experimental and the control groups. The maximum hip extension attained for the subjects of the experimental group was significantly lower than that of the control group (Table 3). The three children with spastic diplegic cerebral palsy remained in hip and knee flexion (crouch gait) throughout the complete cycle, never reaching hip extension. The cycle analyzed extended from the moment of contralateral foot contact (left foot), through backward swing of the right lower extremity, and up to the full contact of the right foot. To compensate for the difficulties in extending the hip, these subjects rotated the pelvis and trunk, to use the separation of the lower extremities (decreasing the hip adduction) as a mechanism to step backwards. The inability for the subjects with spastic diplegic cerebral palsy to walk backwards with Iong steps may be due to deficits in the extensor control of the hip and lower extremities, which makes it difficult for the individual to counteract the strong flexor activity of the hips and 
knees plus the force of gravity, or due to the presence of contractures of hip adductors and hamstrings which impedes the necessary separation of the lower extremities during backward walking.

The three children with spastic diplegia showed a pattern of increased hip flexion, especially at the beginning of the cycle, considerably higher than the control group, probably needed to clear the foot and avoid touching the floor (see Figures 1 \& 2).

Towards the middle or the end of the cycle, these subjects began to extend the hip, but unlike the control group, they were not able to attain the neutral position of the hip, always remaining in some degree of hip flexion.

In Figure 1, the curves for the experimental group are flatter than those for the control group. The curves for the experimental group show a rapid descent into flexion and a slower movement towards neutral. The control group shows a slower descent into flexion and a faster and greater movement towards neutral and extension.

No significant differences were found between both groups in the amount of hip sagittal range of motion (Table 3), but these motions occurred towards the flexion in the group of children with spastic diplegic 
cerebral palsy and towards neutral and extension in the control group (Figure 1).

The pelvic range of motion data (Table 2) did not show important differences in the amount of range of motion of the subjects from both groups, or in the amount of anterior or posterior tilt found. However, the three subjects with SDCP showed a tendency to rotate the pelvis in anterior tilt during backward walking (Figure 2), while two of the subjects of the control group rotated the pelvis towards posterior tilt (Figure 2).

The magnitude of the EMG activity during backward walking in the child with spastic diplegic cerebral palsy (Table 1) was very high in comparison to the child of same age from the control group ( 92 of MVC for the gluteus maximus and 96 for the biceps femoris versus $37 \%$ and $40 \%$ respectively). This data may suggest that, in order for the subject with spastic diplegic cerebral palsy to walk backwards, he/she must recruit "almost all" the motor units available in the system.

Limitations and Problems of the study This pilot study encountered several problems 
during its execution. The degree of cooperation on the part of young children with and without disabilities was overestimated, especially during the assessment of electromyographic activity. This fact limited the EMG data collected in the experimental group. The uniform application of markers and surface electrodes in all subjects was difficult and it may have affected the accuracy of the kinematic data collected.

The experimental group of subjects was not chosen at random, and although it included ambulatory and non-ambulatory subjects from ages 4 to 12 , it is not representative of the total population of children with spastic diplegic cerebral palsy.

It was also difficult to gather the maximum voluntary contraction (MVC) data in the experimental group, which consisted of children with voluntary and involuntary motor control problems, as well as subnormal intellectual abilities. MVC in this population should be carefully interpreted. In one of the subjects from the experimental group the best MVC was not obtained in an isolated contraction of the muscle group being tested, but from the response of the whole lower extremity (total extensor pattern). This is probably something to consider in future research with this population of children. During the execution of this study, an effort was 
made in order to maintain uniformity with regard to step length and gait speed in both groups, parameters that may alter the kinematics and the EMG activity data. In spite of this, the data of the experimental group represents a slower gait, shorter backward steps, and a tendency to remain in double stance, and required intensive verbal cuing during testing.

During this study, the subjects walked backwards a minimum of three times. From these trials, the selection of a "typical" cycle in one of the trials was subjectively chosen and analyzed. For further research, it may be necessary to define this "typical" cycle quality in operational terms. 
Conclusions and Benefits of the Study

This study found that children with spastic diplegic cerebral palsy (SDCP) walked backwards in a more flexed pattern, attaining less maximum hip extension than the control group. The three subjects also rotated the pelvis anteriorly during backward walking. This tends to suggest that independent backward walking is not an effective way to promote active hip extension in this population, and that clinicians are challenged to research the clinical assumption that the facilitation of hip extension in assisted backward walking may result in increased active extension during forward gait.

This study also found that SDCP subjects needed a higher percentage of their maximum voluntary contraction (MVC) to walk backward, suggesting that they used "almost all" the motor units available in their system. It may be argued than MVC is not an appropriate measurement in subjects with voluntary or involuntary motor control disorders.

Although this study was not directed to measure gait speed, the data suggested that children with SDCP walked backward more slowly, took shorter steps and tended to spend more time in double stance. 
They also tended to rotate the body and use the knee flexion as "compensatory mechanisms" to make up for the insufficient hip extension.

Further studies may be necessary for clinicians to determine ways to assist or facilitate hip and knee extension during backward walking, as well as to increase the step length and gait speed. Transfer of learning studies may determine if these assistive techniques do result in active motor learning for these subjects. 


\section{References}

Aicardi, J., \& Bax, M. (1992). Cerebral palsy. In J. Aicardi (Ed), Diseases of the nervous system in

Childhood (pp. 330-374). New York: Cambridge University Press.

Basmajian, J. V., \& De Luca , C. J. (1985). Muscles alive : Their functions revealed by

electromyography. (5th ed.). Baltimore: Williams and Wilkins.

Bobath, B. (1975 a) Adult hemiplegia: Evaluation and treatment. (pp.110-111). London: William Heineman Medical Books.

Bobath, B. (1975 b) Motor development in the different types of cerebral palsy. London: William Heinemann Medical Books.

Caplan, F., \& and Caplan T. (1977). The second twelve months of life. (p 300), NY : Grosset and Dunlap. Cipriani, D. J., Armstrong, C. W., \& Gaul, S. (1993) .

Backward walking at three levels of treadmill inclination : An EMG and kinematics analysis. Physical Therapy, (Annual Conference Abstracts, Abstract No PO R097-7). Gage, J., Fabian, D., Hicks, R., \& Tashman, S. (1984). Pre and post-operative gait analysis in patients 
with spastic diplegia: A preliminary report. Journal of Pediatric Orthopedics, Vol 4, (6) , 715-724.

Greiner, B. M., (1993). Gait parameters of children with spastic diplegia: A comparison of effects of posterior and anterior walkers. Arch Phys Med Rehabil, 74 (4) pp.381-385.

Hagberg, B., Hagberg, G., \& Vonwendt, L. (1989). The changing panorama of cerebral palsy in Sweden.V: The birth year period 1979-1982. Acta Paediatrica Scandinavica, 78, 282-290.

Howell-Garvey, V., Tylkowski, C., Kates, C. \& Miller, G. J. (1987). The influence of type of walker on the gait of children with cerebral palsy. Dev Med and Child Neur, 29 (Supl.55), 5 .

Logan, L., Byers-Hinkley, K., \& Ciccone, C. (1987). Anterior vs posterior walkers for children with cerebral palsy: a gait analysis study. Proceedings of the 1987 Annual meeting of the American Academy for Cerebral Palsy and Developmental Disabilities (pp.21). Philadelphia: J.B.Lippincott.

Kadada, M. P., Wooten, M. E., Gainey , J., \& Cochran, V. B. (1985). Repeatability of phasic muscle activity: Performance of surface and intramuscular wire electrodes in gait analysis. Journal of Orthopaedic Research, 3,350-359. 
Lockart, R. D., Hamilton, G. F. \& FYfe, F. W. (1965). Anatomy of the human body (pp.297-299). Philadelphia J.B. Lippincott.

Nelson, K. B., Ellenberg, J. H. (1978). Epidemiology of cerebral palsy. In B.S. Shoenberg (Ed.) Advances in neurology, (Vol 19, pp.421-435), New York: Raven Press.

Nene, A. V., Evans, G. A. \& Patrick, J. H. (1993). Simultaneous multiple operations for spastic diplegia. Outcome and functional assesment of walking in 18 patients. Journal of Bone and Joint Surgery of Great Britain, 75 (3) $488-494$.

Perry, J. \& Antonelli, D. (1993). Electromyography in gait analysis. The standard, Oxford Metrics Ltd, Oxford, England.

Sutherland, D. H. (1982). Gait disorder in childhood and adolescence, (pp. 129-141). Baltimore: Williams and Wilkins.

Sutherland, D. H., Olshen, R., Biden, E., \& Wyatt, M. (1988) . The Development of Mature Walking.

Philapelphia: J.B. Lippincott.

Tylkowski, C. (1988). The Influence of hamstring and hip flexor musculature on crouch gait in spastic cerebral palsy as determined by gait analysis. Proceedings of the 1988 Annual meeting of the American Academy for Cerebral Palsy and 
Developmental Medicine, (pp.14-15). Philadelphia: J.B.Lippincott .

Tylkowski, C., \& Howell, V. (1990, August). Gait study (No 516-05,11,15). Presented at the course Basics in Computerized Gait Analysis, Miami,FL.

Tylkowski, C., \& Howell, V. (1993). Gait analysis report, Miami Children's Hospital, Miami, FL. Winter, D. A. (1987). The biomechanics and motor control of human gait. Waterloo, Ontario: University of Waterloo Press. Walthard, K. M. and Tchicaloff, M. (1971). Motor points. In S. Licht (Ed), Electrodiagnosis and electromyography (pp. 153-170) New Haven, Connecticut: Elizabeth Licht. 